\title{
Macrocephaly in infancy: benign enlargement of the subarachnoid spaces and subdural collections
}

\author{
Jacqueline Tucker, ${ }^{1}$ Arabinda Kumar Choudhary, MD, ${ }^{2,4}$ and Joseph Piatt, MD ${ }^{3,5}$ \\ ${ }^{1}$ Pennsylvania State University, State College; Departments of ${ }^{4}$ Radiology and ${ }^{5}$ Neurological Surgery, Sidney Kimmel Medical \\ College, Thomas Jefferson University, Philadelphia, Pennsylvania; and ${ }^{2}$ Department of Radiology and ${ }^{3}$ Nemours Neuroscience \\ Center, Alfred I. duPont Hospital for Children, Wilmington, Delaware
}

\begin{abstract}
OBJECTIVE Benign enlargement of the subarachnoid spaces (BESS) is a common finding on imaging studies indicated by macrocephaly in infancy. This finding has been associated with the presence of subdural fluid collections that are sometimes construed as suggestive of abusive head injury. The prevalence of BESS among infants with macrocephaly and the prevalence of subdural collections among infants with BESS are both poorly defined. The goal of this study was to determine the relative frequencies of BESS, hydrocephalus, and subdural collections in a large consecutive series of imaging studies performed for macrocephaly and to determine the prevalence of subdural fluid collections among patients with BESS.
\end{abstract}

METHODS A text search of radiology requisitions identified studies performed for macrocephaly in patients $\leq 2$ years of age. Studies of patients with hydrocephalus or acute trauma were excluded. Studies that demonstrated hydrocephalus or chronic subdural hematoma not previously recognized but responsible for macrocephaly were noted but not investigated further. The remaining studies were reviewed for the presence of incidental subdural collections and for measurement of the depth of the subarachnoid space. A 3-point scale was used to grade BESS: Grade 0, < $5 \mathrm{~mm}$; Grade 1, 5-9 mm; and Grade 2, $\geq 10 \mathrm{~mm}$.

RESULTS After exclusions, there were 538 studies, including 7 cases of hydrocephalus (1.3\%) and 1 large, bilateral chronic subdural hematoma (0.2\%). There were incidental subdural collections in 21 cases (3.9\%). Two hundred sixtyfive studies (49.2\%) exhibited Grade 1 BESS, and 46 studies (8.6\%) exhibited Grade 2 BESS. The prevalence of incidental subdural collections among studies with BESS was 18 of $311(5.8 \%)$. The presence of BESS was associated with a greater prevalence of subdural collections, and higher grades of BESS were associated with increasing prevalence of subdural collections. After controlling for imaging modality, the odds ratio of the association of BESS with subdural collections was $3.68(95 \% \mathrm{Cl} 1.12-12.1, p=0.0115)$. There was no association of race, sex, or insurance status with subdural collections. Patients with BESS had larger head circumference Z-scores, but there was no association of head circumference or age with subdural collections. Interrater reliability in the diagnosis and grading of BESS was only fair.

CONCLUSIONS The current study confirms the association of BESS with incidental subdural collections and suggests that greater depth of the subarachnoid space is associated with increased prevalence of such collections. These observations support the theory that infants with BESS have a predisposition to subdural collections on an anatomical basis. Incidental subdural collections in the setting of BESS are not necessarily indicative of abusive head injury.

http://thejns.org/doi/abs/10.3171/2015.12.PEDS15600

KEY WORDS abusive head injury; benign enlargement of the subarachnoid spaces; BESS; hydrocephalus; macrocephaly; subdural collection

$\mathrm{M}$ ACROCEPHALY is a very common reason for referral of infants to neurosurgical office practices. The great majority of infants with macrocephaly have either constitutional megalencephaly or benign enlargement of the subarachnoid spaces (BESS). Also known as "external hydrocephalus," BESS is, with rare exceptions, a transient developmental phenomenon characterized by a disproportion between the volume of the brain and the volume of the skull, with deep subarachnoid spaces accounting for the difference. Because BESS does

ABBREVIATIONS BESS = benign enlargement of the subarachnoid spaces; SSFSE = single-shot fast spin echo; US = ultrasound SUBMITTED October 7, 2015. ACCEPTED December 7, 2015.

INCLUDE WHEN CITING Published online March 4, 2016; DOI: 10.3171/2015.12.PEDS15600. 
not require treatment, it has not received investigative attention in proportion to its prevalence. Indeed, its prevalence is poorly defined.

Conditions that cause macrocephaly and require treatment are less common. The 2 major considerations are hydrocephalus and chronic subdural hematoma. Experience suggests that these conditions ought to be identifiable on a clinical basis. Infants with BESS are usually well, unless they exhibit mild gross motor delays related to the size of their heads. Infants with hydrocephalus are expected to exhibit fullness of the fontanelle, at least, even if they are not yet symptomatic or developmentally delayed, whereas infants with constitutional megalencephaly or BESS typically have slack fontanelles. Infants with macrocephaly due to chronic subdural hematoma usually are chronically ill with failure to thrive and developmental delay, and they often come to attention with full fontanelles and seizures. The sensitivity, specificity, and predictive values of these generalizations have never been subjected to quantitative scrutiny, and evidence-based guidelines for imaging of infants with macrocephaly do not exist. Consequently, the number of infants subjected to imaging for macrocephaly is large, and the diagnostic yield is low.

These circumstances lead to identification of many well infants with BESS, and a small fraction of them are found to have incidental, asymptomatic subdural fluid collections. These collections are presumed to be variable mixtures of blood and CSF, although their composition is rarely confirmed because drainage is seldom indicated clinically. Some of these collections may develop on an anatomical basis related to craniocerebral disproportion, but others may be due to unrecognized or unreported injury. The goal of this study was to determine the relative frequencies of BESS, hydrocephalus, and subdural collections in a large consecutive series of imaging studies performed for macrocephaly and to determine the prevalence of subdural fluid collections among patients with BESS. On the basis of a mechanical explanation for these collections, we hypothesized that infants with collections have larger head circumference Z-scores and higher grades of BESS.

\section{Methods}

Radiology reports of brain ultrasound (US), CT, and MRI studies were subjected to digital text search for the term "macrocephaly." The search extended from May 2009 to May 2015. Studies in patients older than 2 years were excluded. For patients who had multiple brain imaging studies, only the first study performed because of macrocephaly was included. Patients who were determined from review of notes to have had a history of trauma or hydrocephalus prior to the index imaging study were excluded. The study set thus consisted of images obtained because of unexplained macrocephaly. This study was supervised by the Nemours institutional review board.

All images in the study set were reviewed by a senior investigator (a pediatric neurosurgeon) to identify causes of macrocephaly and to search for subdural collections that may have been overlooked in the initial interpretations. Macrocephaly was attributed to hydrocephalus if moderate or marked ventriculomegaly was present, and the diagnosis was confirmed by the responsible clinician in the electronic medical record. Mild degrees of ventriculomegaly in patients who were not treated for hydrocephalus were not considered to be the cause of macrocephaly. Macrocephaly was attributed to chronic subdural hematoma if the collections were diffuse, bilateral, and of sufficient volume to account for enlargement of the head. Other subdural collections were designated "incidental" if they did not displace or deform the brain and if they did not contribute to macrocephaly. Incidental subdural collections were confirmed by review of the other senior investigator (a pediatric neuroradiologist).

Measurement of the subarachnoid space was used to grade BESS. Measurements were performed digitally using the tool provided in Philips iSite Enterprise. In axial images, the measurements were taken over the frontal lobes in cuts passing through the bodies of the lateral ventricles. In coronal images, measurements were made over the convexities dorsal to the sylvian fissures in cuts passing through the bodies or frontal horns of the lateral ventricles. Measurements of the subarachnoid space were taken from the crest of a gyrus to the nearest point on the inner table of the skull. For each study, the largest such measurement that the grader could identify was considered to be the depth of the subarachnoid space (Fig. 1). BESS was graded as follows: Grade $0,<5 \mathrm{~mm}$; Grade 1, 5-9 mm; and Grade 2, $\geq 10 \mathrm{~mm}$. Grading was performed by 1 investigator (J.T.). A random selection of 100 studies was graded by the other 2 investigators for assessment of interrater reliability.

Associations between categorical variables were assessed using Fisher's exact test, the chi-square test, or the Cochrane-Mantel-Haenszel test. Quantitative variables were compared using t-tests or generalized linear modeling. Interrater reliability in the diagnosis of BESS and its grading was analyzed with a kappa statistic. Data were organized in Excel 2013, and statistical analysis was conducted using SAS 9.4 (SAS Institute, Inc.).

\section{Results}

Among the 538 imaging studies that met inclusion criteria, 7 studies (1.3\%) demonstrated hydrocephalus and 1 study $(0.2 \%)$ demonstrated a large, symmetrical, chronic subdural hematoma. Three hundred eleven studies (57.8\%) exhibited BESS. In 265 studies (49.2\%), BESS was Grade 1 , and in 46 studies (8.6\%), BESS was Grade 2. There were 21 studies $(3.9 \%)$ with incidental subdural collections, only 10 of which were recognized in the initial radiological interpretations. The association of degrees of BESS with subdural collections is presented in Table 1.

The prevalence of incidental subdural collections among studies with BESS was 18 of $311(5.8 \%)$. There was a strong association between the prevalence of subdural collections and the grade of BESS ( $p<0.0001)$. Subdural collections were more prevalent among studies with any degree of BESS than among other studies $(p=0.0115)$. Subdural collections were more common among studies with Grade 2 BESS than among studies with Grade 1 $\operatorname{BESS}(\mathrm{p}=0.0088)$. 

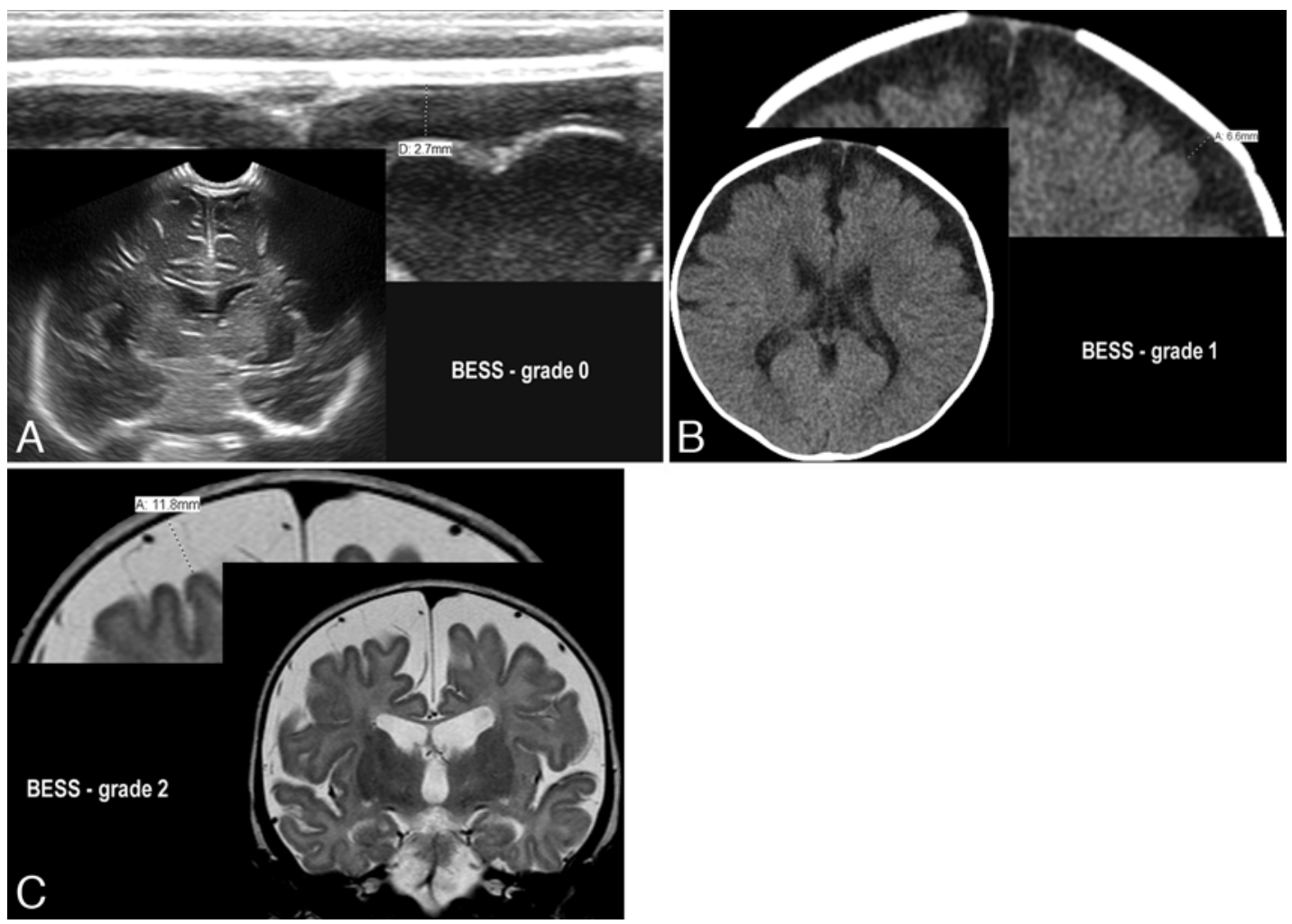

FIG. 1. Grades of BESS and illustrative measurements of the subarachnoid spaces. A: Grade 0 BESS. B: Grade 1 BESS. C: Grade 2 BESS.

Four imaging modalities were represented in this study: US, CT, single-shot fast spin echo (SSFSE) MRI (which is a limited T2-weighted study), and complete MRI. Older patients tended to undergo MRI or SSFSE, and the youngest patients tended to be sent for US. Although no formal comparisons were undertaken, these modalities seemed to differ greatly with respect to sensitivity in detection of subdural collections and reliability in assessment of the depth of the subarachnoid space. The Cochrane-MantelHaenszel test was used to analyze the association of BESS with subdural collections while controlling for imaging modality. The prevalence of subdural collections was 11 of $100(11 \%)$ for MRI, 3 of 85 (3.53\%) for CT, 7 of 277 (2.53\%) for US, and 0 of 68 for SSFSE. The effect of imaging modality having been controlled, there was a significant association between BESS and subdural collections (OR 3.68, 95\% CI 1.12-12.1, p = 0.0115). By this same analytical technique, with imaging modality and the presence of BESS having been controlled, there was no association between race (white or other), sex, or insurance status (commercial or other) and the presence of subdural collections (data not shown).

Contemporaneous head circumference Z-scores were available for 139 patients. Patients with BESS had higher Z-scores than patients who did not have BESS, with means of 1.49 and 0.61 , respectively (95\% CI of the difference $0.27-1.49, \mathrm{p}=0.0051$ ). Patients with incidental subdural collections actually had smaller head circumference $\mathrm{Z}$-scores than other patients, with means of 0.60 and 1.21, respectively, but this difference was not significant (95\% CI of the difference -2.54 to $1.33, \mathrm{p}=0.4958$ ).

There was no association between BESS and patient age (data not shown). Patients with subdural collections were slightly younger than patients without subdural collections, with mean ages of 0.57 and 0.73 years, respectively $(95 \% \mathrm{CI}$ of the difference -0.03 to $-0.30, \mathrm{p}=0.0186)$.

Interrater reliability in the assessment of the presence of BESS was assessed pairwise among all 3 investigators. With respect to the presence or absence of BESS, kappa statistics were $0.68,0.68$, and 0.61 . With respect to the grading of BESS, weighted kappa statistics were 0.62 , 0.60 , and 0.50 . None of the $95 \%$ CIs for these comparisons included 0 .

Of the 10 cases that were recognized to exhibit incidental subdural collections in the initial interpretations, only 3 patients were evaluated by the hospital's child abuse

TABLE 1. Association of incidental subdural collections with grades of BESS*

\begin{tabular}{cc}
\hline Grade of BESS & Incidental Subdural Collections (\%) \\
\hline 0 & 3 of $219(1.4)$ \\
\hline 1 & 11 of $265(4.2)$ \\
\hline 2 & 7 of $46(15.2)$ \\
\hline Total & 21 of $530(4.0)$ \\
\hline
\end{tabular}

${ }^{*} \chi^{2}=19.2060, \mathrm{df}=2 ; p<0.0001$. 
consultant. Only 1 patient, who had the largest of the incidental subdural collections in this series, was reported to a state agency. This child had negative results on dilated funduscopic examination and skeletal survey.

Subsequent brain imaging was performed for 7 patients with incidental subdural collections. After intervals ranging from 3 to 24 months, all collections resolved without surgical treatment.

\section{Discussion}

Images were selected for this study with the goal of surveying the findings among infants with macrocephaly. A text search of radiology records proved not to be entirely specific. Some infants were not actually macrocephalic. On review, some infants were determined to be victims of trauma even though the radiology request simply stated "macrocephaly." In cases of hydrocephalus and subdural collections, the electronic medical record was reviewed to exclude patients known to have had these conditions before imaging, but the medical record was not reviewed for the other 530 cases. Thus the prevalence rates of various findings in the current series may have limited external validity and must be considered illustrative only. Of note was the low prevalence of actionable findings such as hydrocephalus and chronic subdural hematoma, which had a combined rate of only $1.5 \%$. Brain imaging for macrocephaly in infancy has almost negligible diagnostic yield. The findings in the current study necessarily reflect local practice patterns, but they illustrate a need for evidencebased imaging guidelines.

Although BESS is common in pediatric neurosurgical practices, it is not as familiar to other pediatric practitioners. There are only a few estimates of its prevalence in the literature. Among 20 patients subjected to MRI for macrocephaly, Alper et al. found 13 cases (65\%) of BESS. ${ }^{1}$ Greiner et al. reviewed 168 imaging studies performed because of macrocephaly for children 24 months of age or younger; BESS was present in 108 of them (64\%). ${ }^{4}$ In a clinical study of 88 young children with macrocephaly, Medina et al. identified 66 patients $(75 \%)$ with "idiopathic external hydrocephalus." 9 The current study seems to be the largest ever to have addressed the prevalence of BESS. The lower prevalence of BESS reported here, 57.8\%, may reflect contamination of the study group by cases of infants who were not really macrocephalic and thus may be considered a lower limit on the actual prevalence.

The association of increasing degrees of BESS with increasing prevalence of subdural collections supports the concept of an anatomical predisposition to subdural fluid collection among infants with this form of craniocerebral disproportion. A graded association has not been reported before. Papasian and Frim developed a simple mathematical model to demonstrate increasing vulnerability of bridging convexity veins to stretch and rupture with increasing depth of the subarachnoid space. ${ }^{11}$ This model does not explain why hemorrhage from ruptured veins collects in the subdural space instead of the subarachnoid space. Nor does it explain collections that are predominantly CSF. A more elaborate finite element analysis by Raul et al. reached the opposite conclusion-that bridging veins are subject to less strain in infants with BESS..$^{12}$ Predisposition to subdural hemorrhage is a familiar neurosurgical phenomenon among older adults with brain atrophy. Extending this expectation to infants with BESS is a very short conceptual leap, but the pathomechanical details remain obscure.

The prevalence of BESS acquires forensic significance in the context of child abuse. ${ }^{14}$ Subdural hemorrhage in infancy without a verifiable history of trauma is widely judged to be suggestive (or even diagnostic) of abusive head injury, but this generalization disregards volume and composition. Several older case series of BESS mention examples of small subdural collections of CSF, blood, or a mixture of these 2 in asymptomatic children., ${ }^{2,3,5,10,13} \mathrm{Be}-$ cause of small numbers and variable methods of ascertainment, no credible estimate of the prevalence of subdural collections among infants with BESS can be developed from this older literature. Nor can these cases be cleared retrospectively of the suspicion of unrecognized abuse.

McNeely et al. excluded cases of abuse from their review of BESS at the Montreal Children's Hospital. Over a 6-year period, they identified 7 cases of BESS with subdural collections. ${ }^{8}$ Unfortunately, they did not report the total number of cases of BESS or estimate the prevalence of subdural collections. More recently, in the study by Greiner et al., among 108 infants with BESS, 6 cases (5.6\%) had subdural collections, and review of hospital records indicated that 2 of the 6 had been reported to state agencies for suspicion of abuse. ${ }^{4}$ McKeag et al. found 4 examples of subdural collections among 177 imaging studies of infants with macrocephaly featuring BESS (2.3\%). One of the 4 children had rib fractures and the case was reported to child protective services?

In the current series, 1 patient was found to be macrocephalic because of large, bilateral chronic subdural hematomas. After complete evaluation, that patient was believed to be the victim of abuse and was referred to the appropriate agency. Only 10 of the 21 incidental subdural collections were recognized prospectively; 3 were evaluated for abuse, and 1 was reported to a state agency. Contemporary authors acknowledge that incidental subdural collections in infancy are not necessarily indicative of abusive head injury. However, in the absence of a reliable rule for excluding the possibility of abuse, authors who express an opinion recommend investigation. ${ }^{4,7,14,15}$ Whether-or how frequently-small, incidental collections evolve into large, symptomatic collections is another question of forensic concern. Incidental subdural collections resolved without treatment in all cases that had follow-up imaging in this series, but the small number of such cases does not support general conclusions.

Interrater agreement in identification and grading of BESS in the current study was only fair. The kappa statistic is a common metric of agreement, but its interpretation is not very intuitive. A defined amount of agreement between 2 raters can be expected on the basis of chance alone. The kappa statistic reflects the fractional location of the observed agreement on the interval between agreement by chance and total agreement. Kappa values between 0.50 and 0.68 indicate that the grading of BESS has construct validity, but better agreement is desirable 
for research purposes. We followed other authors in our methodology for measurement of the subarachnoid space and in acceptance of $4 \mathrm{~mm}$ as an upper limit of normal for its depth over the convexities, but we are unaware of any previous analysis of interrater reliability. ${ }^{6,8}$ Future studies of BESS will benefit from automated digital segmentation technologies for objective measurement of the volumes of intracranial spaces.

\section{Conclusions}

Imaging for macrocephaly in infancy has very low yield in identification of conditions that require treatment. BESS, a condition that does not require treatment, is very common among infants with macrocephaly. Incidental subdural collections are associated with both the presence and the degree of BESS. Incidental subdural collections in infancy are not necessarily indicative of abuse, but the prevalence of abuse among such cases is not known.

\section{Acknowledgments}

Ms. Tucker was supported by the Nemours Summer Undergraduate Research Program.

\section{References}

1. Alper G, Ekinci G, Yilmaz Y, Arikan C, Telyar G, Erzen C: Magnetic resonance imaging characteristics of benign macrocephaly in children. J Child Neurol 14:678-682, 1999

2. Azais M, Echenne B: [Idiopathic pericerebral swelling (external hydrocephalus) of infants.] Ann Pediatr (Paris) 39:550-558, 1992 (Fr)

3. Gout A, Gautier I, Bellaiche M, Pinard JM, Tremon M, Rodriguez D, et al: [Idiopathic peri-cerebral enlargement in infants: simple anatomical variant or hemorrhagic risk factor?] Arch Pediatr 4:983-987, 1997 (Fr)

4. Greiner MV, Richards TJ, Care MM, Leach JL: Prevalence of subdural collections in children with macrocrania. AJNR Am J Neuroradiol 34:2373-2378, 2013

5. Laubscher B, Deonna T, Uske A, van Melle G: Primitive megalencephaly in children: Natural history, medium term prognosis with special reference to external hydrocephalus. Eur J Pediatr 149:502-507, 1990

6. Libicher M, Tröger J: US measurement of the subarachnoid space in infants: normal values. Radiology 184:749-751, 1992

7. McKeag H, Christian CW, Rubin D, Daymont C, Pollock AN, Wood J: Subdural hemorrhage in pediatric patients with enlargement of the subarachnoid spaces. J Neurosurg Pediatr 11:438-444, 2013
8. McNeely PD, Atkinson JD, Saigal G, O'Gorman AM, Farmer JP: Subdural hematomas in infants with benign enlargement of the subarachnoid spaces are not pathognomonic for child abuse. AJNR Am J Neuroradiol 27:1725-1728, 2006

9. Medina LS, Frawley K, Zurakowski D, Buttros D, DeGrauw AJ, Crone KR: Children with macrocrania: clinical and imaging predictors of disorders requiring surgery. AJNR Am J Neuroradiol 22:564-570, 2001

10. Mori K, Sakamoto T, Nishimura K, Fujiwara K: Subarachnoid fluid collection in infants complicated by subdural hematoma. Childs Nerv Syst 9:282-284, 1993

11. Papasian NC, Frim DM: A theoretical model of benign external hydrocephalus that predicts a predisposition towards extra-axial hemorrhage after minor head trauma. Pediatr Neurosurg 33:188-193, 2000

12. Raul JS, Roth S, Ludes B, Willinger R: Influence of the benign enlargement of the subarachnoid space on the bridging veins strain during a shaking event: a finite element study. Int J Legal Med 122:337-340, 2008

13. Ravid S, Maytal J: External hydrocephalus: a probable cause for subdural hematoma in infancy. Pediatr Neurol 28:139141,2003

14. Vinchon M, Delestret I, DeFoort-Dhellemmes S, Desurmont M, Noulé N: Subdural hematoma in infants: can it occur spontaneously? Data from a prospective series and critical review of the literature. Childs Nerv Syst 26:1195-1205, 2010

15. Wittschieber D, Karger B, Niederstadt T, Pfeiffer H, Hahnemann ML: Subdural hygromas in abusive head trauma: pathogenesis, diagnosis, and forensic implications. AJNR Am J Neuroradiol 36:432-439, 2015

\section{Disclosures}

The authors report no conflict of interest concerning the materials or methods used in this study or the findings specified in this paper.

\section{Author Contributions}

Conception and design: Piatt. Acquisition of data: all authors. Analysis and interpretation of data: Piatt. Drafting the article: Piatt. Critically revising the article: Piatt, Choudhary. Reviewed submitted version of manuscript: all authors. Approved the final version of the manuscript on behalf of all authors: Piatt. Statistical analysis: Piatt. Administrative/technical/material support: Piatt. Study supervision: Piatt.

\section{Correspondence}

Joseph Piatt, Division of Neurosurgery, Nemours Neuroscience Center, Alfred I. duPont Hospital for Children, 1600 Rockland Rd., Wilmington, DE 19803.email: jpiatt@nemours.org. 